\title{
THEORY OF TRANSPORT PROCESSES
}

\author{
J.-P. ZAHN \\ Observatoire Midi-Pyrénées \\ 14, avenue E. Belin \\ \$1400 Toulouse \\ France \\ and \\ Columbia University \\ New York, N.Y. 10027 \\ U.S.A.
}

\begin{abstract}
This review focuses on the transport of matter and angular momentum in the radiative zones of stellar interiors. The two main causes of such transport are the convective overshooting in the vicinity of convection zones, and the slow motions (meridional circulation and turbulence) due to the rotation of the star. In addition, momentum can be transfered through waves (generated by the motions above) and through magnetic stresses. The characteristics of those processes are examined, with special emphasis on turbulent diffusion.
\end{abstract}

\section{Introduction}

Transport processes play a key role in the structure and the evolution of a star: above all, they deliver the energy which is produced in the deep interior to the surface, from where it is radiated in into space. Quite naturally, the two modes of transport which are responsible for this, namely radiative transfer and thermal convection, are considered by the astrophysicists as two major subjects, and they occupy a prominent place both in research and in teaching.

The purpose of this review to examine some other processes which contribute, in stellar radiation zones, to the transport of matter and angular momentum. It is not necessary to explicit here in detail the motivations for such particular interest: they have been outlined in the introductory talk by $\mathrm{E}$. Schatzman. Let us just recall that some chemical abundances at the surface of the Sun can only be interpreted by invoking some transport of matter in the radiative interior (Schatzman 1969, 1977; Schatzman and Maeder 1981). And the internal rotation of the Sun, which is being unveiled by the thrilling results of helioseismology, is also the result of the transport of angular momentum within the radiation zone.

Let us begin by a brief inventory of the transport processes that are likely to occur. One can distinguish two classes among them: the microscopic processes, which operate at the particle level, and the macroscopic ones, which involve motions on larger scales. 


\subsection{MICROSCOPIC PROCESSES}

Microscopic diffusion is caused by the collisions between particles, and by their interactions with the radiation field. In a stellar interior, the ambiant medium is non uniform, due to the stratification of density and temperature; therefore, the interactions between particles of different species tend to separate them. Similarly, the slight anisotropy of the radiation field also to produces a chemical composition gradient.

The theory of such processes has been established by Chapman and Cowling (1970), and their application to stellar envelopes has been developed mainly by Michaud (1970) and his co-workers; an excellent review on the subject has been written by Vauclair and Vauclair (1982).

Thermal conduction in a gas is also due to particle collisions, through which thermal kinetic energy is exchanged; it is very sirailar to microscopic diffusion.

Radiative transfer is by far the most powerful transport process in a stellar radiation zone, at least as long as matter is non degenerate. Except in the vicinity of the surface, stellar matter is optically thick; radiation and matter interact so strongly that they achieve the so-called local thermodynamic equilibrium (LTE). Then the radiation field becomes very nearly isotropic, and radiative transfer can be treated likewise as a diffusive process.

The governing equation for all those diffusion processes is

$$
\frac{\partial c}{\partial t}=\nabla \cdot D \nabla c+\{s\}
$$

where $c$ is the quantity being diffused (energy, concentration of chemical species, etc.) and $\{S\}$ the sources and sinks of that quantity. Various notations are used for the diffusion coefficient, in order to distinguish between the different processes when they compete; here it is simply labeled $D$. Let us recall that in LTE, the radiative diffusivity is related to the opacity $\kappa$ through

$$
K=\frac{4 a c T^{3}}{3 C_{P} \rho^{2} \kappa},
$$

with the usual notations for the physical constants and for the temperature, the density and the specific heat.

Viscous friction also can be considered as a diffusive process, in that case of momentum, the diffusivity then being the viscosity $\nu$. One of the main characteristics of stellar interiors is the great disparity between that diffusion of momentum and the radiative transport of energy: the ratio $\operatorname{Pr}=\nu / K$, which is called the Prandtl number, is extremely small (typically $10^{-6}$ or less). In contrast, the fluids which are the most familiar to us, such as air or water, have a Prandtl number of order unity.

\subsection{MACROSCOPIC PROCESSES}

Advection through large scale circulations is the simplest of macroscopic processes, and we have many examples of it around us, in Nature and in our household (central heating!). If $V$ represents the velocity field, the governing equation for the advection of a scalar quantity $c$ just expresses the conservation of that quantity:

$$
\frac{\partial c}{\partial t}+\mathbf{V} \cdot \nabla c=\{S\}
$$


with the same notations as above.

In radiative interiors, two causes of large scale circulations have been identified. One has been extensively studied: it is the thermal imbalance of a rotating star. When submitted to the centrifugal force, a star can no longer achieve radiative equilibrium, and this induces a meridional advection of heat which is known as the Eddington-Sweet circulation (Eddington 1925, Vogt 1925, Sweet 1950, Mestel 1953, Kippenhahn 1958, McDonald 1972, Tassoul and Tassoul 1982, etc.)

Much less attention has been paid so far by the astrophysicists to the so-called Ekman circulation, which is generated in the boundary layer connecting two regions rotating at different speeds (Ekman 1905; see also Pedlosky 1979). Such a circulation is likely to occur at the bottom of the solar convection zone, in which a strong differential rotation is maintained by the convective motions, whereas the radiation zone below appears to rotate much more uniformly (Dziembowski et al. 1989, Brown et al. 1989). The penetration of such Ekman circulation into the convection zone has already been considered by Bretherton and Spiegel (1968).

One of the consequences of meridional circulation is the advection of angular momentum, which modifies the internal rotation rate of the star, which in turn alters the large scale motion. A complete, physically consistent description of this complex feed back is still lacking; it would require to also include the transport processes which will be discussed next.

Turbulent diffusion. When the velocity field has small scale, time-dependent component $\mathbf{u}$, which may be considered as turbulent (in an intuitive sense - a proper definition of this term is beyond our scope here), the advection equation (2) can be expanded in the following way, by taking suitable averages:

$$
\frac{\partial c}{\partial t}=\nabla \cdot D_{t} \nabla c+\{\text { higher order terms }\}+\{s\} .
$$

For the justification of this procedure we refer to Knobloch (1978); in many instances, it suffices to retain the first term of this formal expansion, which is the second order operator explicited here. The turbulent diffusivity $D_{t}$ can be deduced from the properties of $\mathbf{u}$, as we shall do later. We shall devote the next section to this transport process, since it is likely to play a key role in stellar radiation zones.

In stars, thermal convection is the most efficient form of turbulent diffusion. It is not confined to the convection zone (defined as the region of nearly adiabatic stratification), since the turbulent motions penetrate somewhat into the adjacent layers. This convective overshooting is discussed by J. Massaguer (this volume).

Transport through waves. Due to their oscillatory nature, waves do not contribute much to the transport of matter, unless they reach a rather large, finite amplitude (Weiss and Knobloch 1989). But they are very efficient in transporting energy and momentum, and therefore they too deserve a more detailed examination (in section 3 ). 


\section{Turbulent diffusion}

\subsection{ESTIMATE OF THE TURBULENT DIFFUSIVITY}

We have already stated that, to first approximation, the transport in a turbulent medium of a scalar quantity (such as temperature, chemical species, etc.) can be described as a diffusion process. For simplicity, we assume here the velocity field to be isotropic enough so that the turbulent diffusivity reduces to a scalar, $D_{t}$. This coefficient is determined by the characteristics of the turbulence:

$$
D_{t}=\frac{1}{3} u \ell
$$

where, to first approximation, $u$ is the velocity and $\ell$ is the size of the largest eddies (or, equivalently, the mean free path of those eddies, or mixing length).

It may seem a simple matter to estimate those quantities $u$ and $\ell$, but the prescriptions which are used for that purpose vary from case to case.

For the mixing length $\ell$, one can often take the dimension of the turbulent region, when the largest eddies are of that size. A more refined recipe, due to Prandtl, is to choose the distance to the nearest boundary. But in some instances the kinetic energy is injected at a scale which is smaller than that of the whole unstable region; the most vigorous eddies, which contribute most to the turbulent transport, are then of intermediate size.

In the strong stratification of a stellar convection zone, it is not clear whether the eddies extend (or travel) over large vertical distances. It is customary then to follow E. Vitense (1953), and to relate the mixing length to the local pressure scale height, $\ell=\alpha H_{P}$, the coefficient $\alpha$ being calibrated by comparing the numerical models with the observations.

To estimate the velocity $u$, various prescriptions are available. Let us take for example the turbulent shear flow: $u$ is then of the order of the variation $\Delta U$ of the mean flow speed over the considered domain. The simplest case is when the differential velocity is maintained by some external force at a constant level $\Delta U$, as it occurs in Couette flows. But often that $\Delta U$ is governed by the strength of the turbulence, which in turn is determined by another condition, such as the momentum flux or the heat flux which has to be carried. Sometimes it is possible to estimate the rate $\varepsilon_{t}$ at which kinetic energy is injected into the turbulent motions at the scale $\ell$, from where it cascades down to smaller scales, to be dissipated there through viscous friction. In this case, if one further assumes that the turbulent eddies obey the Kolmogorov law (see Landau and Lifshitz 1987), $u$ can be derived from

$$
\varepsilon_{t} \approx u^{3} / \ell
$$

An alternate approach is to estimate $u$ through the growth rate $1 / \tau$ of the considered instability :

$$
u \approx \ell / \tau
$$

this conditions implies that the growth of the instability saturates when the non-linear term of the momentum equation, $u \nabla u$, reaches the same level as the linear terms.

The growth rate $1 / \tau$ is often approximated by the growth rate derived from the linear perturbation theory, for lack of something better. But it is more correct to calculate it, whenever it is feasible, in the non-linear regime which is attained by the instability. This is 
done for instance in the classical mixing-length theory for convection, where the velocity is estimated by imagining an eddy accelerated in the actual superadiabatic density gradient:

$$
u^{2}=C_{1} \frac{g}{H_{P}}\left(\nabla_{r a d}-\nabla_{a d}\right) \ell^{2}
$$

The expression for the (prescribed) convective flux is then

$$
F_{c}=C_{2} \rho u^{3} \frac{H_{P}}{\ell} ;
$$

the constants $C_{1}$ and $C_{2}$ are numerical coefficients of order 1 , depending on phenomenological details of that approach. Notice that one retrieves here the same ratio $u^{3} / \ell$ as in eq. 5 above: it is equivalent to specify the convective flux or the kinetic energy injection rate.

\subsection{TURBULENT DIFFUSION IN STELLAR RADIATION ZONES}

A radiation zone is a region of stable thermal stratification; therefore, the convective instability that causes the turbulent motions which are suspected there cannot be ascribed to the convective instability (except in the vicinity of a convection zone, from which there is some penetration into the stable layers).

What may then be the cause of such turbulence? It turns out that many instabilities are likely to occur in a radiation zone; they have been described in several reviews (see for instance Knobloch and Spruit 1982, 1983, or Zahn 1983). Such instabilities convert into turbulent kinetic energy other forms of energy which is stored in the star (thermal energy, gravitational, magnetic, kinetic energy of large scale flows, etc.)

These instabilities compete with each other, and it is the strongest of them that will control the turbulent transport in the radiation zone. In all likelihood, the most powerful are the shear instabilities due to differential rotation, since they are of dynamical nature and therefore have the fastest growth-rate.

Some amount of differential rotation is always present in a stellar radiation zone. It is due to several causes: contraction or expansion of the star while it evolves, angular momentum loss through a wind, coupling with a differentially rotating convection zone, meridional circulation, tidal braking in a binary star.

Let us examine those shear instabilities in more detail. For the discussion, it is convenient to distinguish between vertical differential rotation, in which the angular velocity varies with depth, and horizontal differential rotation, where it varies with latitude.

2.2.1. Vertical differential rotation. A typical velocity profile likely to be encountered in the vertical direction, for instance below the convection zone of a solar-type star, is the socalled mixing layer: the angular velocity adjusts from a constant value $\Omega$ above to a higher value $\Omega+\Delta \Omega$ below, within a layer of thickness $L$. Since the profile presents an inflexion point, the flow is unstable to infinitesimal perturbations (Rayleigh 1880); the instability is of dynamical type, and its growth rate is of order

$$
\tau^{-1} \approx \Delta \Omega_{s} / L
$$

$s$ being the distance to the rotation axis (the viscosity has been neglected, for it plays here a negligible role). 
But in a stratified medium such as stellar interior, the buoyancy force acts to hinder the instability. If there were no radiative damping, the instability would be suppressed for

$$
\Delta \Omega s / L<N,
$$

$N$ being the buoyancy frequency

$$
N^{2}=\frac{g}{H_{P}}\left(\nabla_{a d}-\nabla_{r a d}\right)
$$

However, radiative damping smoothes out the temperature differences, and therefore it lowers the threshold of the instability, which occurs then as soon as

$$
(\Delta \Omega R / L)^{2}>N^{2} \operatorname{Pr} R_{c} .
$$

In this criterion $R_{c}$ is the critical Reynolds number associated with that profile (it is of order 100), and $\mathrm{Pr}$ is the Prandtl number (ratio between the viscosity $\nu$ and the thermal diffusivity $K$ ), which in a radiation zone is of order $10^{-6}$ or less.

This condition is valid as long as the star has a uniform chemical composition. If there is a (stable) gradient of molecular weight, the buoyancy force is only partly weakened through radiative damping, and one recovers the original criterion (eq. 8), the buoyancy frequency being then reduced to

$$
\left(N_{\mu}\right)^{2}=\frac{g}{H_{P}} \frac{\mathrm{d} \ln \mu}{\mathrm{d} \ln P} .
$$

In all cases where the instability occurs, the largest turbulent eddies have a size of order $L$ and a velocity of order $\Delta \Omega s$, and therefore the turbulent diffusivity is of order

$$
D_{t} \approx \Delta \Omega s L \text {. }
$$

Those two criteria (eq. 9 and 10) can be extended to smoother velocity profiles, which do not exhibit an inflexion point, although the instability is then of different nature as we shall see next; all it needs is to replace the finite difference $(\Delta \Omega / L)$ by the derivative $\partial \Omega / \partial r$.

2.2.2. Horizontal differential rotation. When the rotation rate varies with latitude, the situation presents two main differences with the vertical differential rotation that we have just considered.

First, the instability of a horizontal shear flow cannot be hindered by a stratification, since the buoyancy force acts only in the vertical direction. And it can been shown that the Coriolis force has no influence either on the instability criterion (see Tritton and Davies 1981).

Second, the horizontal velocity profiles that are likely to occur have in general no inflexion point, and therefore they are stable against infinitesimal perturbations. But they are liable to finite amplitude instabilities, as observed in Nature and in the laboratory, provided the Reynolds number reaches some critical value. Work is in progress to determine theoretically the threshold of that instability (see for instance Lerner and Knobloch 1988).

When a horizontal shear flow becomes unstable, it generates eddies which have the same vorticity as the mean flow, and which are therefore horizontal, and two-dimensional. Those billows, in turn, undergo a three-dimensional instability, provided the vertical motions are not hindered by some restoring force. 
One such force is the Coriolis force in the rotating star, which will dominate the dynamics of all eddies for which (see Hopfinger et al. 1982)

$$
u / \ell<\Omega
$$

those will remain horizontal, and they will not contribute to turbulent diffusion in the vertical direction. But the smallest eddies are not sensitive to the Coriolis force; they are three-dimensional, and they obey Kolmogorov's law (eq. 5). Their distribution begins at the scale which verifies both

$$
u^{\prime} / \ell^{\prime} \approx \Omega \text { and }\left(u^{\prime}\right)^{3} / \ell^{\prime} \approx \varepsilon_{t},
$$

and therefore the vertical turbulent diffusivity is given by

$$
D_{t} \approx u^{\prime} \ell \approx \varepsilon_{t} / \Omega^{2} \text {. }
$$

The other restoring force is the buoyancy, but it only operates when there is a vertical gradient of molecular weight, since here again the temperature differences are smoothed out by radiative damping. Such a $\mu$-gradient will inhibit three-dimensional turbulence for the eddies whose turn-over rate is less than the residual buoyancy frequency (eq. 10)

$$
u / \ell<N_{\mu}
$$

and it will suppress it entirely when (see Zahn 1983)

$$
\left(N_{\mu}\right)^{2}>\varepsilon_{t} / \nu \text {. }
$$

A rather small gradient of molecular weight thus suffices to prevent turbulent diffusion in the vertical direction (for instance, that due to the varying composition of ${ }^{3} \mathrm{He}$ in the Sun). However, such a " $\mu$-barrier" (as Mestel called it in an other context) will still allow momentum transport through gravity waves, as we shall see later on.

\subsection{TURBULENT DIFFUSION ASSOCIATED WITH MERIDIONAL CIRCULATION}

We have seen that the strength of the turbulent motions is determined by the energy injection rate $\epsilon_{t}$. As an illustrative example, we shall estimate this rate in the case where the turbulent motions are caused by a meridional circulation.

We have already mentioned that radiative equilibrium can no longer be achieved in a rotating star. From Von Zeipel's famous paradox (1924), Eddington (1925) and Vogt (1925) drew the conclusion that the star must be the seat of a large scale meridional circulation, such that

$$
\rho T \mathbf{U} \cdot \nabla S=-\nabla \cdot F,
$$

with $S$ being the specific entropy and $\mathbf{F}$ the radiation flux. The procedure to determine the velocity $U$ was established by Sweet (1950); the circulation time may be expressed in terms of the Kelvin Helmholtz time $t_{K H}=G M^{2} / L R$ and of the oblateness due to the centrifugal force

$$
t_{c i r c} \approx t_{K H}\left(\frac{\Omega^{2} R^{3}}{G M}\right)^{-1}
$$


That meridional circulation advects angular momentum, whose conservation requires

$$
\frac{\partial}{\partial t} s^{2} \Omega+U \cdot \nabla\left(s^{2} \Omega\right)=\Gamma
$$

where $\Gamma$ is the torque exerted per unit mass by the turbulent motions (as above, $s$ is the distance to the rotation axis). Likewise, we may express the rate of variation of the rotational energy:

$$
\frac{\partial}{\partial t} \frac{1}{2}(s \Omega)^{2}+\Omega U \cdot \nabla\left(s^{2} \Omega\right)=\Omega \Gamma .
$$

In a stationary state, the advection term balances the right hand side, which is the work done by the turbulent torque, and therefore the energy injection rate into the turbulence. Splitting the angular velocity into its mean and fluctuating parts (over a level surface) $\Omega(r)+\delta \Omega(r, \theta)$, and subtracting the kinetic energy of the mean flow from that which is advected into the layer, we obtain the following expression for the turbulent energy input, averaged over a horizontal layer (Zahn 1987):

$$
\epsilon_{t}(r)=-\int_{0}^{1} \delta \Omega(r, \theta) \mathbf{U} \cdot \nabla\left(\Omega_{0}(r) \sin ^{2} \theta\right) d(\cos \theta) .
$$

Let us stress that this expression is valid for any type of meridional circulation, either the Eddington-Sweet or the Ekman circulation. But from now we must specificy which of those we are considering, since we have to provide the value of the large scale velocity.

When dealing with the Eddington-Sweet circulation, a crude approximation of that energy generation rate $\varepsilon_{t}$ is

$$
\varepsilon_{t} \approx C \frac{L}{M}\left(\frac{\Omega^{2} R^{3}}{G M}\right)^{2}
$$

but for most applications it is necessary to use the full equation 20 , with suitable expressions of $\mathbf{U}$ and $\delta \Omega$.

One knows how to derive the meridional velocity from the rotation law $\Omega(r, \theta)$; for instance, when the star is homogeneous and the departures from solid rotation are not too large, the vertical component of $\mathbf{U}$ takes the simple form $U_{r}(r, \theta)=-U_{2}(r) P_{2}(\cos \theta)$, $U_{2}(r)$ being a positive function of $r$ (McDonald 1972).

The difficulty comes from the poor knowledge of the differential rotation $\delta \Omega(r, \theta)$, which is governed by the horizontal transport of angular momentum, through the two-dimensional eddies mentioned above, and also through the internal waves. The modelization of this transport is still an unsolved problem. In the meanwhile, all we can do is to assert $\delta \Omega=-C \Omega_{0} P_{2}(\cos \theta)$, introducing a coefficient $C$ to be calibrated with the observations, much as the mixing-length parameter $\alpha$ used to model stellar convection zones.

This type of turbulent diffusion appears to play a major role in the depletion of $\mathrm{Li}$ in solar-like stars (Baglin et al. 1985, Vauclair 1988). It may also affect the abundance of $\mathrm{Li}$ observed in the old halo stars (Vauclair 1988). 


\section{Transport by waves}

\subsection{GENERAL PROPERTIES}

Waves occur in any continuous medium which is in stable equilibrium, due to the very existence of a restoring force. The waves encountered in a stellar radiation zone are of several types, depending on the forces that come into play:

\begin{tabular}{cc} 
Restoring force & Type of waves \\
\hline pressure & acoustic, p-modes \\
buoyancy & internal, g-modes \\
Coriolis & inertial \\
id. with curvature & Rossby, toroidal \\
Laplace-Lorentz & Alfvén
\end{tabular}

This classification assumes that only one restoring force is operating, but often there are two, or more, giving rise to mixed modes (magneto-acoustic waves, for example).

A familiar property of waves is that they transport energy, but they also transport momentum, and thus angular momentum. In fact, the quantity which is conserved in a travelling wave is the action $E / \sigma$

$$
\frac{\partial}{\partial t}\left(\frac{E}{\sigma}\right)+\nabla \cdot\left\{\left(\mathbf{U}+\mathbf{c}_{g}\right)\left(\frac{E}{\sigma}\right)\right\}=\{S\}
$$

( $E$ : energy density; $\sigma$ : local frequency; $\mathrm{U}$ : velocity of the ambiant medium; $\mathbf{c}_{g}$ : group velocity).

To study the transport through wave motion, one has to follow the whole history of the wave: How is it generated? How does it propagate? Where is it damped? Is it destroyed? (In other words: where does the wave release what it transports?)

Here we shall focus only on the two first families of waves; for lack of results bearing on stellar interiors, we skip the waves due to the Coriolis force. The magnetic coupling through Alfvén waves will be treated by $H$. Spruit (this volume).

\subsection{WAVE TRANSPORT IN A STELLAR RADIATION ZONE}

3.2.1. Acoustic waves. The most powerful source for wave production is thermal convection: as observed on the Sun, a substantial fraction of the turbulent convective energy is converted into acoustic waves. And some of those waves penetrate into the deep interior, as demonstrated by the frequencies and the wave-lengths that are detected by the helioseismological techniques. However they are only slightly damped in the radiative core, due to their short period, and therefore they deposit there very little of the energy (and momentum) which is carried by them. One still lacks of a quantitative treatment of this transport, to check whether it is indeed negligible. 
3.2.2. Gravity waves. It is expected that internal gravity waves are excited in the overshoot region just below the convection zone, although there is no observational proof of this yet. The periods and wave-lengths of such waves may be infered from the kinematic properties of the lower convection zone (which are model dependent).

It turns out that those internal waves, due to their long period, are severely damped through radiative dissipation (Press 1981); hence they do not penetrate far into the radiation zone, although they may be very effective in coupling the rotation of that region with that of the convection zone.

However, those long period waves will survive in spite of the strong radiative damping if there is a vertical gradient of molecular weight: although the temperature fluctuations are smoothed out, buoyancy will then still operate on the density fluctuations due to the chemical inhomogeneities. In other words, such a $\mu$-gradient can be the site for isothermal gravity waves.

Internal waves are likely to be generated also by the shear instabilities which have been described above, especially when there is a gradient of molecular weight. This is suggested in particular by an experience performed by Stillinger et al. (1983), who studied the turbulence induced by a grid in a stably stratified fluid. They found that the dynamics of the large scales is dominated by the buoyancy force and that they take the form of internal waves; only the smaller scales, for which $u / \ell>N_{\mu}$ (see eq. 14), participate in what they call "active turbulence", which is responsible for the vertical diffusion of matter. Those internal waves may thus carry energy and momentum through a region which is impermeable to turbulent diffusion; in other words, angular momentum is transported, but chemicals are not.

Another interesting property of gravity waves has been discussed by Goldreich and Nicholson (1989) in the context of tidal braking in binary stars: in a differentially rotating star, those waves may dump their energy and momentum at the corotation point. To be specific, the frequency $\sigma$ of the wave, which is $\omega$ in the rest frame, is a function of depth in the local frame, which rotates at the depth-dependent velocity $\Omega(r)$ :

$$
\sigma=m(\Omega(r)-\omega),
$$

where $m$ is the azimuthal wave-number. At corotation, both the phase velocity $\sigma$ and the group velocity vanish, and the vertical wavenumber $k_{r}$, which obeys

$$
\sigma k_{r}=N m / r
$$

becomes infinitely large. Thus the wave either breaks there, or it is strongly damped.

\section{Conclusion}

To summarize, some turbulent diffusion is likely to occur in the radiation zone of any star; it is caused by the instabilities due to the ever present differential rotation. But this type of transport operates in the vertical direction only when the composition gradient is not too large. Presently, the weak point of the theory is the poor knowledge of the horizontal transport of angular momentum, which determines the rate at which kinetic energy is transferred from the differential rotation of the star into the turbulent motions.

On the other hand, transport by waves is a promising mechanism to explain the redistribution of angular momentum within a star, without affecting its chemical composition. 
Unfortunately, very little is known so far about the efficiency of this process, although it is clear that it too will be linked with the energy generation rate characterizing the turbulent motions which are responsible for the production of such waves.

Finally, it appears that two regions of a star like the Sun play a key role in those transport processes: the layers located just below the convection zone, where nearly all such processes are likely to operate and to compete (convective overshoot, meridional circulation, turbulent diffusion, waves), and the upper slope of the ${ }^{3} \mathrm{He}$ abundance, which inhibits the vertical diffusion of matter.

\section{References}

Bretherton, F.P. and Spiegel, E.A. 1968, Astrophys. J. 153, 277

Brown, T.M., Christensen-Dalsgaard, J., Dziembowski, W.A., Goode, P., Gough, D.O. and Morrow, C.A. 1989 Astrophys. J. 343, 526

Chapman, S. and Cowling, T.G. 1970, The Mathematical Theory of Non-uniform Gases (Cambridge Univ. Press)

Dziembowksi, W.A., Goode, P.R. and Libbrecht, K.G. 1989, Astrophys. J. 337, L53

Ekman, V.W. 1905, Arkiv Matem. Astron. Fysik 2, 11

Eddington, A.S. 1925, Observatory 48, 78

Goldreich, P.A. and Nicholson, P. D. 1989 Astrophys. J., 342, 1075

Hopfinger, E.J., F.K. Browand and Y. Gagne 1982, J. Fluid Mech. 125, 505

Kippenhahn, R. 1958, Z. Astrophys. 46, 26

Knobloch, E. 1978, Astrophys. J. 225, 1050

Knobloch, E. and H.C. Spruit 1982, Astron. Astrophys. 113, 261

Knobloch, E. and H.C. Spruit 1983, Astron. Astrophys. 125, 59

Landau, L. and E. Lifschitz 1987, Fluid Mechanics (English translation, 2nd edition; Pergamon edit., London)

Lerner, J. and Knobloch, E. 1988, J. Fluid Mech. 189, 117

McDonald, B.E. 1972, Astrophys. Space Sci. 19, 309

Mestel, L. 1953, Montly. Not. Roy. Astron. Soc. 113, 716

Michaud, G. 1970, Astrophys. J., 160, 641

Pedlosky, J. 1979, Geophysical Fluid Dynamics (Springer edit.; Berlin, Heidelberg, New York), 482

Press, W. H. 1981, Astrophys. J., 245, 303

Rayleigh, Lord 1880, Scientific Papers, 1, 474 (Cambridge Univ. Press)

Schatzman, E. 1969, Astrophys. Lett. 3, 139

Schatzman, E. 1977, Astron. Astrophys. 56, 211

Schatzman, E. and A. Maeder 1981, Astron. Astrophys. 96, 1

Stillinger, D.C., K.N. Helland and C.W. Van Atta 1983, J. Fluid Mech. 131, 91

Sweet, P.A. 1950, Montly. Not. Roy. Astron. Soc. 110, 548

Tassoul, J.-L. and M. Tassoul 1982, Astrophys. J. Suppl. 49, 317

Tritton, D.J and P.A. Davies 1981, Hydrodynamical Instabilities and the Transition to Turbulence, edit. H.L. Swinney and J.P. Gollub (Topics in Applied Physics; Springer; Berlin, Heidelberg, New York)

Vauclair, S. and Vauclair, G. 1982, Ann. Rev. Astron. Astrophys. 20, 37

Vauclair, S. 1988, Astron. Astrophys. (in press) 
Vitense, E. 1953, Z. Astrophys. 32, 135

Vogt, H. 1925, Astron. Nachr. 223, 229

Von Zeipel, H. 1924, Monthly. Not. Roy. Astron. Soc. 84, 665

Weiss, J.B. and Knobloch, E. 1989, Phys. Rev. A 40, 2579

Zahn, J.-P. 1983, Astrophys. Processes in Upper Main Sequence Stars (Publ. Observatoire Genève), 253

Zahn, J.-P. 1987, The Internal Solar Angular Velocity (eds. B. R. Durney and S. Sofia; Reidel), 201 